\title{
First Principles Calculations of Electronic Band Structure of Nb-Doped ZnO
}

\author{
Hongsheng Zhao ${ }^{1, \mathrm{a}_{1},}$, Nan Zhang ${ }^{1, \mathrm{~b}}$ and Dong Yang ${ }^{1, \mathrm{c}}$ \\ ${ }^{1}$ School of Physics and Electrical Information, Ningxia University, Yinchuan 750021, P.R. China \\ ahszhao.cn@gmail.com, bzhangnan12234492@163.com, cyangdong47924499@163.com
}

Keywords: Nb-doped $\mathrm{ZnO}$; first principles; electronic structure

\begin{abstract}
Based upon the density functional theory (DFT) in this paper, the first-principles approach is used to study the electronic band structure of $\mathrm{Nb}$-doped wurtzite $\mathrm{ZnO}$ with different concentration. Three doped structures were considered: $\mathrm{A} \mathrm{Nb}$ atom replaced by a $\mathrm{Zn}$ atom and two $\mathrm{Nb}$ atoms replaced by two $\mathrm{Zn}$ atoms in different positions. For the pure $\mathrm{ZnO}$, the Fermi level is in the valence band maximum, but in the $\mathrm{Nb}$-Doped $\mathrm{ZnO}$ supercell, Fermi level shifts to the conduction band and exhibits similar metallic properties.
\end{abstract}

\section{Introduction}

$\mathrm{ZnO}$ is an oxide semiconductor and a promising material for electronic, it has become interested topic due to its direct wide bandgap $(\mathrm{Eg} \approx 3.3 \mathrm{eV})[1]$. Compared with $\mathrm{MgO}$ and other optoelectronic materials, $\mathrm{ZnO}$ has a low dielectric constant, big photoelectric coupling rate and excellent piezoelectric. And the exciton binding energies of $\mathrm{ZnO}$ is up to $60 \mathrm{meV}$, it is a Blu-ray emission in the ultraviolet and promising areas of new optoelectronic materials. So it has a wide range of applications in gas sensors[2], ultraviolet lasers[3] and transparent conductive films[4].

In recent years, people in addition to extensive research $\mathrm{ZnO}$ films, also studied the doped $\mathrm{ZnO}$ films of different metals. Doped $\mathrm{Al}[5], \mathrm{Be}[1] \mathrm{In}[6]$ and other elements can be get an ideal electrical properties of $\mathrm{n}-\mathrm{ZnO}$ thin films, significantly improved the conductivity of $\mathrm{ZnO}$ thin films[7]. However, concerning the theoretical calculation and analysis of the influence of the structure of $\mathrm{Nb}$-doped $\mathrm{ZnO}$ were seldom reported.

In this paper, first-principles density functional theory[8] is used to calculate the electronic band structure of $\mathrm{Nb}$-doped $\mathrm{ZnO}$. Four different models are used to study the role of $\mathrm{Nb}$-doped $\mathrm{ZnO}$ (concentration of $\mathrm{Nb}$ are $0,6.25 \%$ and $12.5 \%$ ), and to study band structure and density of states (DOS) of Nb-doped $\mathrm{ZnO}$.

\section{Models and calculation method}

The ideal $\mathrm{ZnO}$ has a hexagonal wurtzite structure. A $2 \times 2 \times 2$ supercell of the wurtzite $\mathrm{ZnO}$ is shown in Fig. 1. Each unit cell contains $2 \mathrm{Zn}$ atoms and $2 \mathrm{O}$ atoms, and there are 32 atoms in all. When one of $\mathrm{Zn}$ atom was replaced by $\mathrm{Nb}$ atom, it is called $\mathrm{Nb}$-doped $\mathrm{ZnO}$. Changed the number of $\mathrm{Nb}$ atoms in the supercell, we can get different concentrations of $\mathrm{Nb}(\mathrm{x}=6.25 \%, 12.5 \%)$. For the concentrations is $\mathrm{x}=6.25 \%$, one model is obtained by replacing $1 \mathrm{Zn}$ atom with $1 \mathrm{Nb}$ atom. Two different positions of $\mathrm{Nb}$ atoms are considered following the research work of Jia et al.[9-10], which concentration is $\mathrm{x}=12.5 \%$ : the "near" configuration, in which the $\mathrm{Nb}$ atoms in the same unit cell are separated by a single $\mathrm{O}$, and the "far" configuration, in which they are connected via -O-Zn-Obond. Then we considered for the effect of doping concentration on the electronic structure of $\mathrm{Nb}$-doped $\mathrm{ZnO}$.

The calculations are carried out by using the CASTEP package provided by the Material Studios 5.0 by Accelrys. The package is an ab initio quantum mechanics codes based on density functional theory[11]. In the package, the ionic potential is substituted by a pseudo-potential, the electronic wave function is expanded by the plane wave, and the exchange and correlative potential of electronic-electronic interactions are improved by the generalized gradient approximation (GGA)[12]. In our calculation, a $4 \times 4 \times 2$ k-point mesh in the Brillouin zone is used, the cutoff

* Author to whom correspondence should be addressed. Electronic mail: hszhao.cn@gmail.com. 
energy of the plane wave is $370 \mathrm{eV}$, and the calculation precision is set to be $2.0 \times 10-5 \mathrm{eV} /$ atom. The test calculations with higher cutoff energies and denser k-point grids are also performed, and the overall results remain unchanged. Then the electronic structures are calculated on the basis of the optimized supercell.

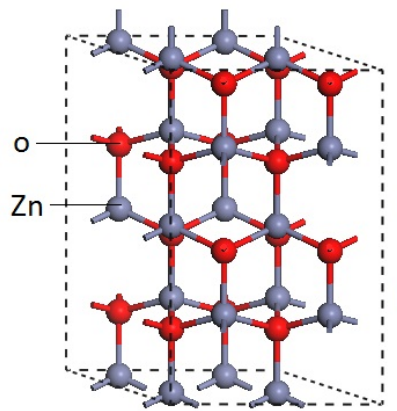

(a)

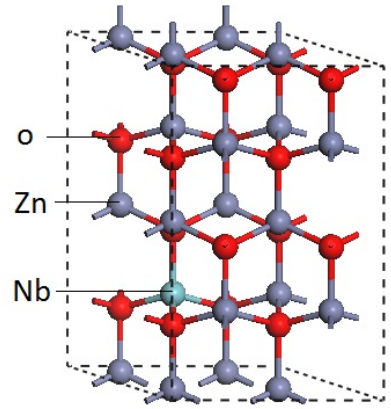

(b)

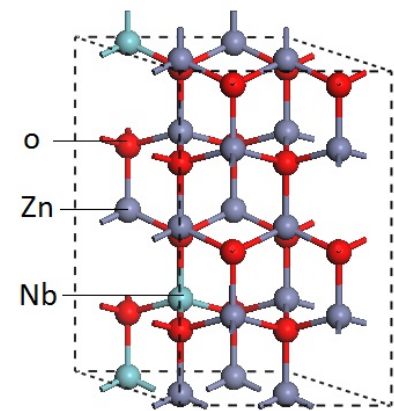

(c)

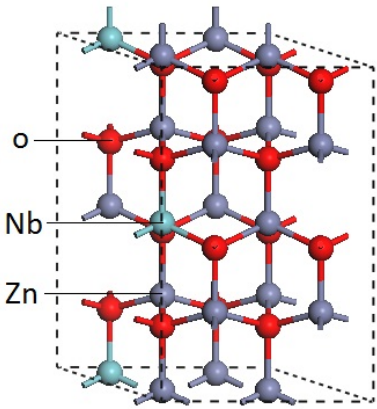

(d)

Fig. 1. (a) pure $\mathrm{ZnO}$, (b) $1 \mathrm{Zn}$ atom replaced $1 \mathrm{Nb}$ atom, $\mathrm{Nb}$-doped $\mathrm{ZnO}$ in the (c) separated by a single $\mathrm{O}$ and $(\mathrm{d})$ separated by $-\mathrm{O}-\mathrm{Zn}-\mathrm{O}-$ bond.

\section{Results and Discussion}

First, the structure of pure $\mathrm{ZnO}$ wurtzite unit cell is optimized in the calculation. The lattice constants are $\mathrm{a}=3.283 \AA$ and $\mathrm{c}=5.311 \AA$. Deviation with the experimental value $(\mathrm{a}=3.253 \AA, \mathrm{c}$ $=5.213 \AA[13])$ is only about $1 \%$, meaning that our calculation is reasonable.

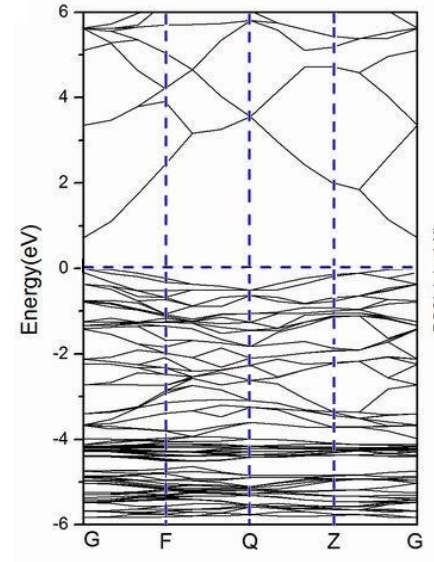

(a)

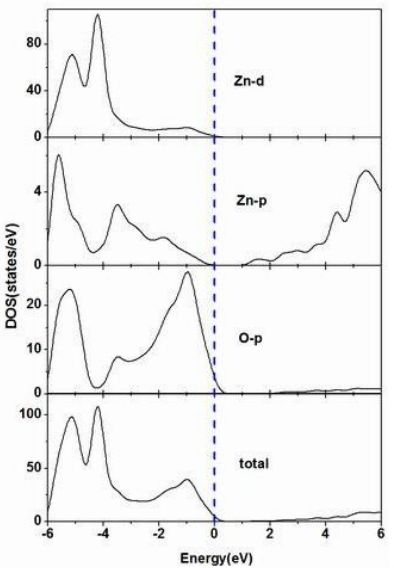

(b)

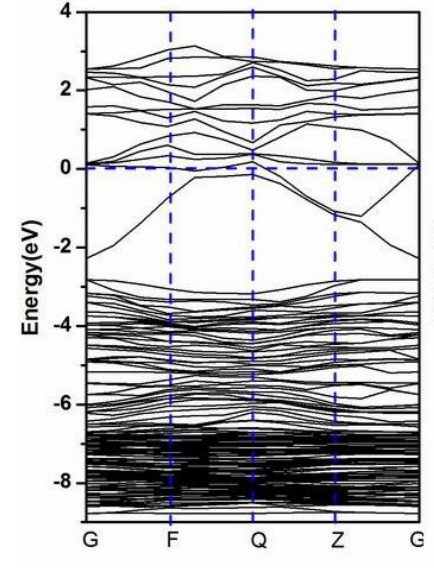

(a)

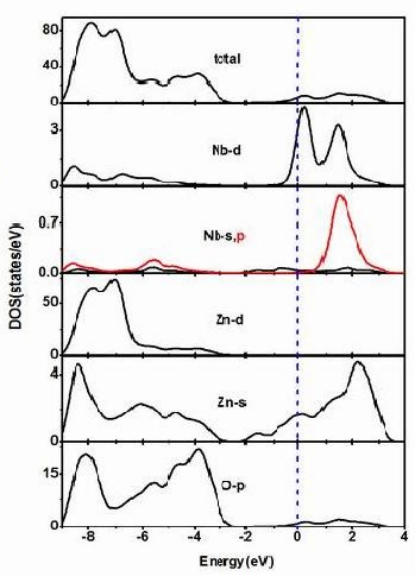

(b)

Fig. 2. (a) Band structure and (b) DOS of pure $\mathrm{ZnO}$.

Fig. 3. (a) Band structure and (b) DOS of $\mathrm{Nb}$-doped $\mathrm{ZnO}$ with $\mathrm{x}=6.25 \%$.

According to the optimized crystal structure, we calculated the band structure, DOS and partial DOS (PDOS)[14] near the Fermi energy of pure ZnO in Fig. 2. From Fig. 2(a), it can be seen that valence band maximum and conduction band minimum are both in the G-point, meaning that pure $\mathrm{ZnO}$ is a semiconductor material with wide direct band gap. Here, the band gap is $0.73 \mathrm{eV}$, which is in agreement with the theoretical results of other researchers[15], but much smaller than the experimental results $(3.3 \mathrm{eV})$. This difference originates from the GGA approximation adopted in the calculation process. From the DOS in Fig. 2(b), we found that the valence band of $\mathrm{ZnO}$ by the $\mathrm{O} 2 \mathrm{~s}$ states occupied the bottom. In the high energy region is mainly composed of electrons of $\mathrm{O}$ $2 p$ states and electrons of $\mathrm{Zn} 3 \mathrm{~d}$ states hybrid. The $\mathrm{O} 2 \mathrm{p}$ states are the most dominant in the energy range between -4 and $0 \mathrm{eV}$, and the $\mathrm{Zn} 3 \mathrm{~d}$ states locate mainly in the energy range between -5.8 and $-4 \mathrm{eV}$. Therefore, the width of the band gap $\mathrm{ZnO}$ mainly decided by $\mathrm{O} 2 \mathrm{p}$ states and $\mathrm{Zn} 4 \mathrm{~s}$ states.

$\mathrm{Next}$, in order to investigate the $\mathrm{Nb}$-doped effect of the electronic structures, we calculated the band structure and DOS of $\mathrm{Nb}$-doped $\mathrm{ZnO}$ with $\mathrm{x}=6.25 \%$ in Fig. 3(a,b). Compared with the pure 
$\mathrm{ZnO}$, the remarkable feature of the band structure of $\mathrm{Nb}$-doped $\mathrm{ZnO}$ is that the forbidden band gap narrowing, which indicates that the material is n-type metal. The upper valence band from -7 to $-3 \mathrm{eV}$ originates mainly from the $\mathrm{O} 2 \mathrm{p}$ states, and the lower valence band from -9 to $-6.4 \mathrm{eV}$ is derived from the $\mathrm{Zn} 3 \mathrm{~d}$ states. The impurity bands of $\mathrm{Nb} 3 \mathrm{~d}$ states lie just across the Fermi energy, which is partially occupied with a bandwidth of $2.5 \mathrm{eV}$. On the other hand, the energy difference between valence band maximum and the Fermi level is about $2.8 \mathrm{eV}$, which is much larger than the band gap of pure $\mathrm{ZnO}(0.73 \mathrm{eV}$ here). As a result, the transition of an electron from valence band to unoccupied states will need more energy in $\mathrm{Nb}$-doped $\mathrm{ZnO}$.

Fig. 4(a, b) shows the DOS of replacing two $\mathrm{Zn}$ atoms with $\mathrm{Nb}$ atoms in the separated by a single $\mathrm{O}$ and separated by -O-Zn-O- bond. From Fig. 4(a), it can be seen that the impurity energy band becomes wider. Meanwhile, along with the concentration of $\mathrm{Nb}$ increased, the band gap became narrower. It determined the width of the band gap energy increased VBM (Valence Band Maximum) and CBM (Conduction Band Minimum) is declining. The energy difference between the valence band maximum and the Fermi level is increased to $2.86 \mathrm{eV}$. Comparing with the results presented in Fig. 4(a, b), the separated by $-\mathrm{O}-\mathrm{Zn}-\mathrm{O}-$ bond makes the $\mathrm{Nb} 3 \mathrm{~d}$ states more localized and strong, and the energy difference between the valence band maximum and the Fermi level becomes larger than the separated by a single $O$, which proves that different doped structure will affect the physical properties of the system, although the configurations are same.

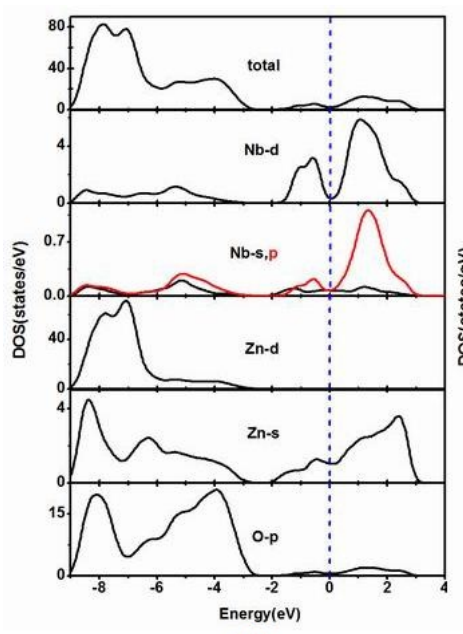

(a)

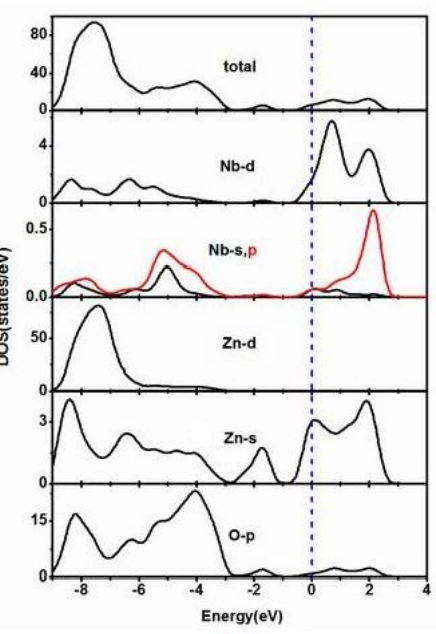

(b)

Fig. 4. $\mathrm{DOS}$ of two $\mathrm{Nb}$-doped $\mathrm{ZnO}$ in the (a) separated by a single $\mathrm{O}$ and (b) separated by -O-Zn-O- bond.

\section{Conclusion}

In this paper, electronic band structure of $\mathrm{Nb}$-doped $\mathrm{ZnO}$ with different situations is studied by using the first-principles with the generalized-gradient approximation. The results showed: $\mathrm{Nb}$-doped $\mathrm{ZnO}$ display n-type semiconductor characteristics. For the pure $\mathrm{ZnO}$, the Fermi level in the valence band maximum. For $\mathrm{Nb}$-doped $\mathrm{ZnO}$, the Fermi level move into conduction band, and to show similar characteristics with the metal.

\section{Acknowledgments}

This work was supported by the National Nature Science Foundation of China (No. 11064009). The authors thank the Ningxia University Supercomputer Center, from where the computational work in this paper was done and the technical support was given. 


\section{References}

[1] Zheng Yongping, C.Z., Lu Yu, Wu Qingyun, Weng Zhenzhen and Huang Zhigao: Journal of Semiconductors Vol. 29 (2008), pp. 2316-2321

[2] He Hongbo, F.Z.: Acta Optica Sinica Vol. 18 (1998), pp. 1676-1680

[3] Hong Ruijin, S.J., He Hongbo, Fan Zhengxiu: Chinese Optics Letters Vol. 3 (2005), pp. 428-431

[4] Zhou Yuming, H.Y., Lu Aixia and Wan Qing: Chinese Physics B Vol. 18 (2009), p. 3966

[5] Huang Yunxia, C.Q., Li Zhimin, Li Guifang, Wang Yupeng, Wei Yunge: ACTA PHYSICA SINICA Vol. 58 (2009), pp. 8002-8006

[6] Chen Kun, F.G., Zhang Yong and Ding Shaofeng: ACTA PHYSICA SINICA Vol. 57 (2008), pp. 3139-3147

[7] Jin Xilian, L.S., Kong Degou, Li Yuncai and DuZuliang: ACTA PHYSICA SINICA Vol. 55 (2006), pp. 4809-4814

[8] Wang Baolin, N.S., Zhao Jijun, Wang Guanghou: Nanotechnology Vol. 18 (2007), p. 345706

[9] Tang Xin, L.H., Ma Chunyu, Zhao Jijun, Zhang Qingyu: ACTA PHYSICA SINICA Vol. 57 (2008), pp. 7807-7813

[10]Lu Yanli, W.H., Hui Liu, Xin Dianliu, Qing Gongsong and Shi Weiren: The Journal of Phsysical Chemistry C Vol. 113 (2009), pp. 8460-8464

[11]Zhang Fuchun, D.Z., Yan Junfeng, Zhang Zhiyong: ACTA OPTICA SINICA Vol. 57 (2008), pp. 3138-3146

[12]Zhang Fangying, Y.J., Zeng Zhi and Zhong Guohua: Chinese Physics Vol. 16 (2007), pp. 3815-3819

[13]De Weichu, Y.P.a.D.L.: Solid State Communications Vol. 143 (2007), pp. 308-312

[14]Duan Manyi, X.M., Zhou Haiping, Chen Qingyun, Hu Zhigang and Dong Chengjun: ACTA PHYSICA SINICA Vol. 57 (2008), pp. 6520-6525

[15]Liu Qijun, Liu Zhengtang, Feng Liping, Xu Bing: Journal of Qingdao University of Science and Technology (Natural Science Edition) Vol. 30 (2007), pp. 377-380 